\title{
DOENÇA DE CROHN: ESTUDO DE CASO
}

\author{
Douglas Rodrigo Cursino dos Santos ${ }^{1}$ \\ Juliana Braga Rodrigues de Castro ${ }^{2}$ \\ Luciana Cursino Parent ${ }^{3}$
}

\section{INTRODUÇÃO}

A doença de Crohn é uma doença inflamatória do trato gastrointestinal que pode se manifestar em qualquer parte do tubo digestivo (desde a cavidade oral até a região anal) sendo mais comum na final do intestino delgado (íleo) e o intestino grosso (cólons). Sua causa ainda não está esclarecida, pois, como não é uma doença contagiosa e pode afetar tanto adultos como crianças, não havendo predominância de sexo. Alguns fatores estão associados ao surgimento da doença e uma maior incidência dentro de núcleos familiares (Io a 25\%) indica importância dos fatores genéticos. Outros fatores tais como o contato com antígenos (vírus e bactérias), fatores ambientais (etilismo, tabagismo, hábitos

alimentares) e emocionais podem representar algum nível de importância em sua apresentação, caracterizando esta doença como multifatorial (ABCD, 2009).

Portanto, não há uma explicação definitiva para a causa da doença, e não há cura descrita, podendo se manifestar ao longo da vida com crises agudas recorrentes, assim como períodos longos de acalmia e ausência dos sintomas, chamado de remissão. $O$ tratamento medicamentoso e/ou cirúrgico pode influenciar positivamente no controle da doença, permitindo longos períodos sem sintomas (SBC, 202I).

\footnotetext{
' Bacharelado em Nutrição - UNIFENAS-MG. Especialização em Nutrição Clínica e Ambulatorial UNYLEYA-DF. Mestrado Profissionalizante em Saúde Coletiva - FACNORTE-PR. Doutorado em Ciências da Educação - FICs - Assunção PY. Docente Integral da Faculdade UNINTA Itapipoca-CE

2 Bacharelado em Nutrição - UECE-CE. Docente Integral da Faculdade UNINTA Itapipoca-CE. Especialização em Residência Multiprofissional em Saúde da Família -Escola de Formação em Saúde da Família Visconde de Sabóia- EFSFVS-SOBRAL-CE. Especialização em Alimentação e Nutrição na Atenção Básica- EAD-ESP-FIOCRUZ-RJ

Mestrado em Nutrição e Saúde - UECE-CE. Docente Integral da Faculdade UNINTA Itapipoca-CE.

${ }^{3}$ Bacharelado em Ciências Biológicas - UFMG-MG. Mestrado em Genética - UFMG-MG

Doutorado em Genética e Melhoramentos de Plantas- ESALQ-USP-SP. Pós doutorado em Genética e Melhoramentos - Universidade de Cornell (Ithaca, NY, EUA). Jephson Science Center, Division of Natural Sciences and Mathematics, Keuka College, Keuka Park, NY, i4478.
} 


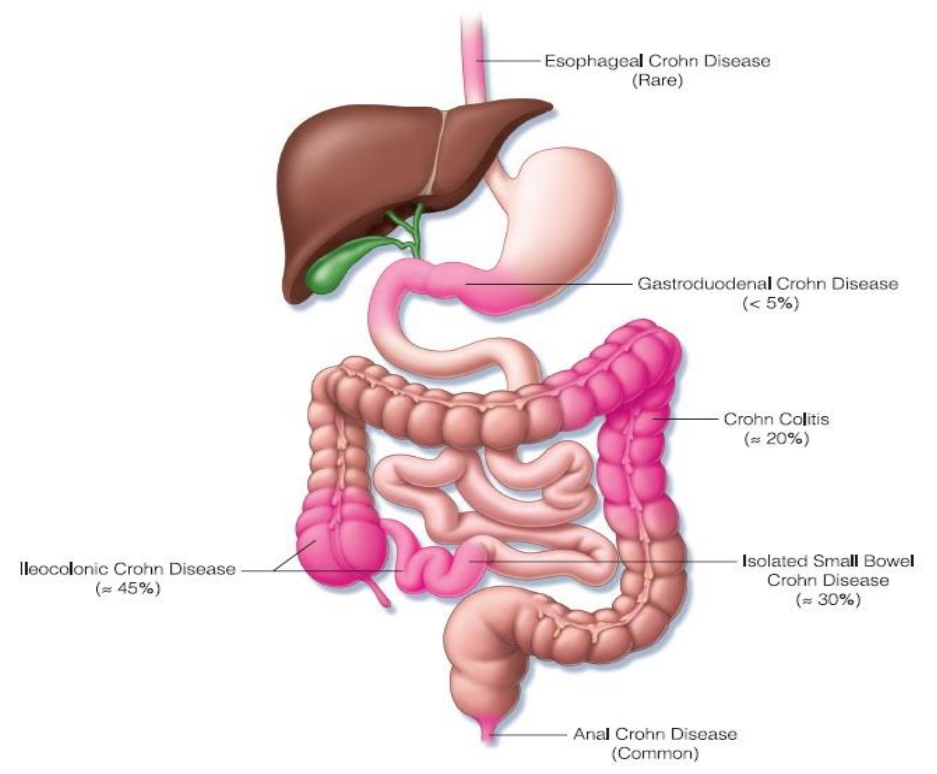

Fonte: CURY \& MOSS, 2015.

A Doença de Crohn (DC) é uma Doença Inflamatória Intestinal (DII) crónica com alguns períodos de agudização, onde as lesões afetam todo o trato gastrointestinal, sendo mais recorrente na região ileocecal. A DC apresenta importantes alterações nutricionais que resultam essencialmente em défices de micronutrientes e desnutrição energético-protéica. A terapia nutricional é avaliada de acordo com a individualidade do paciente com dietas específicas, restrições e suplementação. A Nutrição Enteral (NE) pode ser uma alternativa para fornecer os nutrientes para a recuperação e conservação do estado nutricional. Assim, a manutenção de um bom estado nutricional no paciente com DC revela-se um importante objetivo do seu tratamento de modo a que seja possível proporcionar uma melhor qualidade de vida para o paciente (FLORA et al., 2006).

A Doença de Crohn é frequentemente associada a deficiências nutricionais (DN) severas, apresentando-se o padrão e a severidade da má nutrição dependente da duração, atividade e extensão da doença, onde questões nutricionais relacionadas com a alimentação devem ser consideradas fundamentais na monitorização dos pacientes,sendo a terapêutica planejada individualmente, de acordo com as necessidades do doente. A terapia nutricional oral, enteral ou parenteral pode ser necessária, sendo a via oral a de eleição sempre que esta se mostre adequada e eficaz. Na literatura, verifica-se uma tendência favorável à utilização da Nutrição Enteral (NE), sugerindo unicamente a 
Nutrição parenteral (NP) em situações específicas, ou em que a NE não seja possível. O uso de imunomodeladores tem apresentado uma evolução promissora no tratamento da DC (OLIVEIRA, 2012).

\section{DOENÇA INFLAMATÓRIA INTESTINAL}

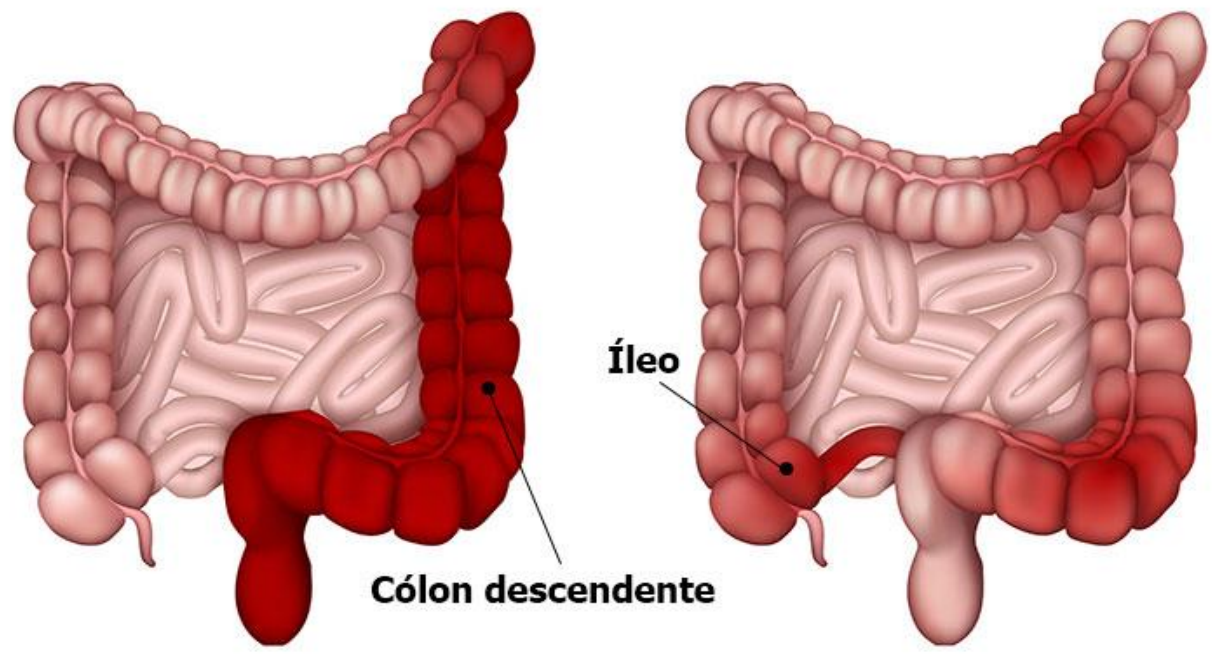

Fonte: CURY \& MOSS, 2015.

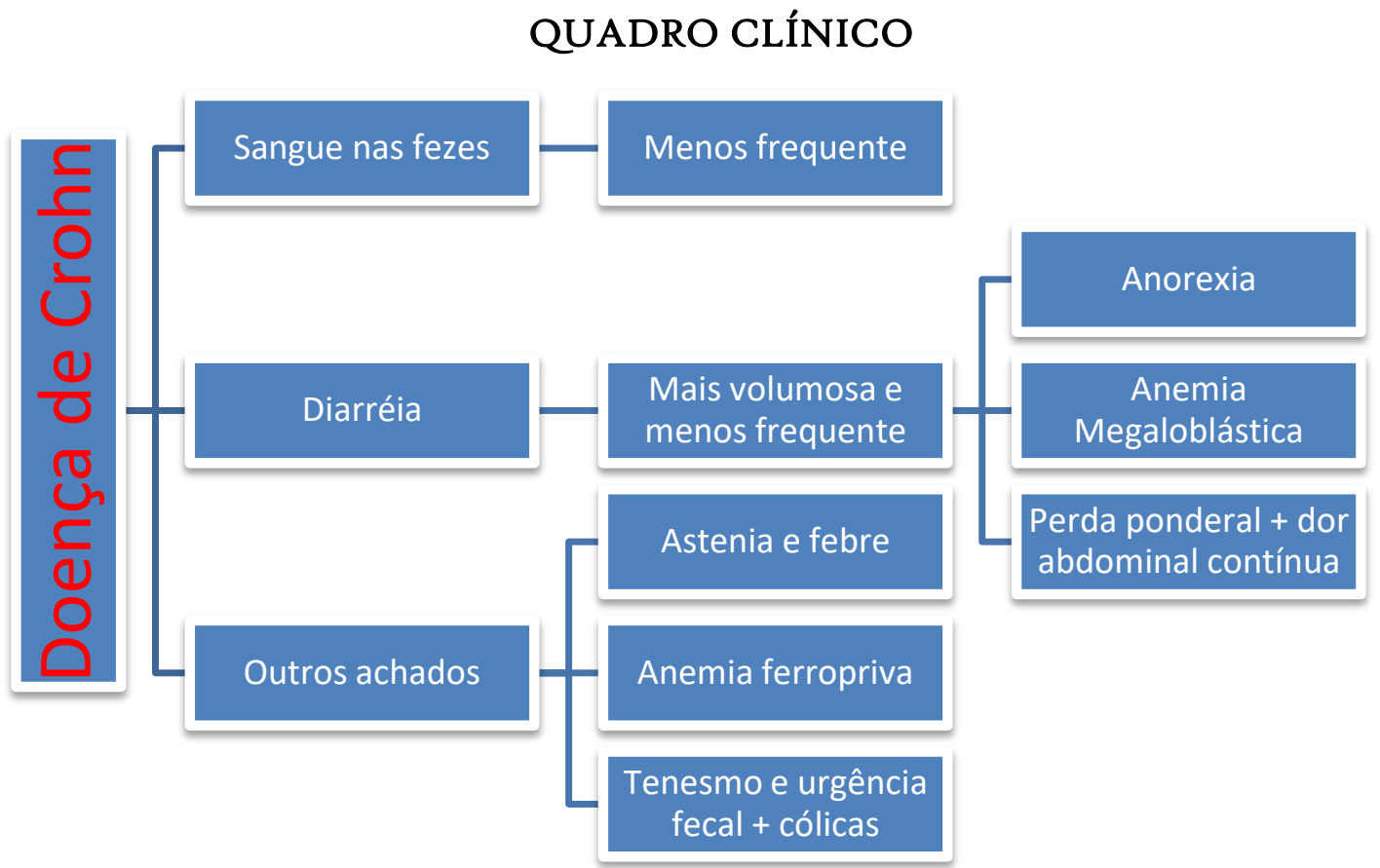

Fonte: Elaborado pelo autor, 2021. 


\section{SINAIS E SINTOMAS}

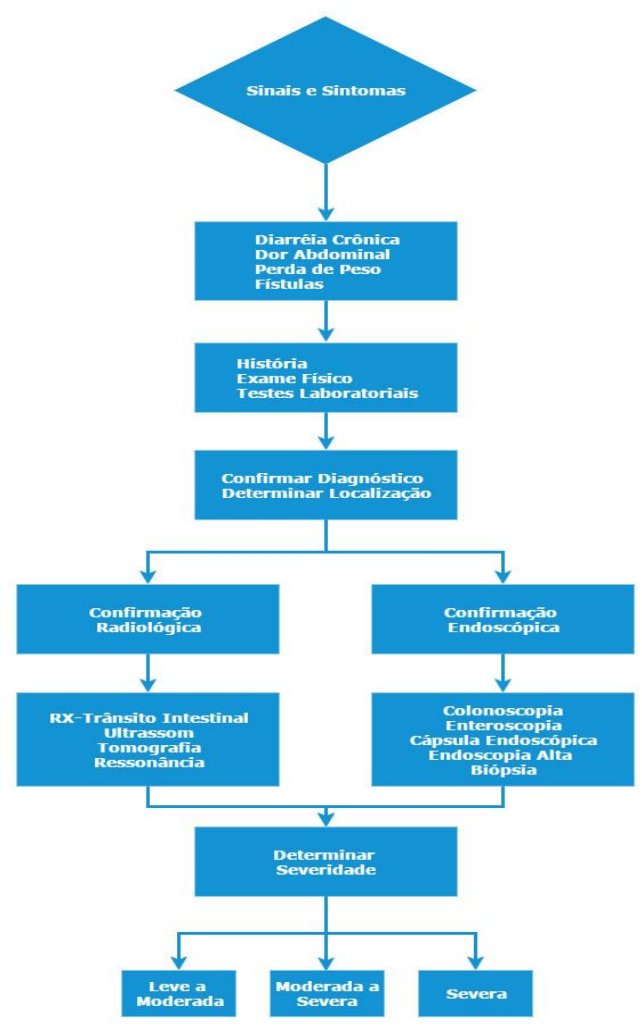

Fonte: Elaborado pelo autor, 2021.

FISIOPATOLOGIA

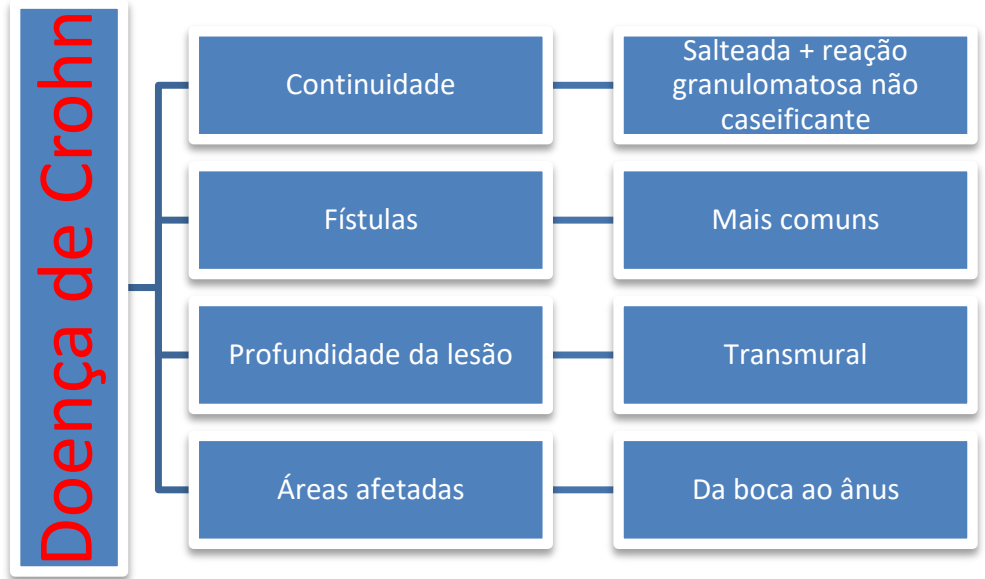

Fonte: Elaborado pelo autor,2021. 


\section{TERAPIA NUTRICIONAL}

Para garantir uma alimentação adequada é um fator importante na DC, sendo o objetivo da intervenção e tratamento nutricional a manutenção da remissão e melhoria do estado nutricional, uma vez que estes pacientes estão em risco de desenvolver desnutrição global e deficiências de nutrientes específicos, o que dificulta os tratamentos e o combate à infeção.Com frequência surgem deficiências nutricionais decorrentes da DC, particularmente quando existe um comprometimento extenso do intestino delgado (LOMER, 20II).

A prevalência de desnutrição proteico-energética está associada a uma ingestão diminuta de nutrientes (devido a anorexia, náuseas, vómitos, dor, desconforto intestinal e uso concomitante de farmacoterapia), má absorção dos mesmos, hipermetabolismo e perdas protéicas a nível intestinal aumentadas, levando a imunodepressão e consequentemente maior probabilidade de ocorrência de complicações infeciosas, atraso no crescimento, osteopénia, má cicatrização, maior risco cirúrgico, redução do trofismo da mucosa intestinal, entre outras. Os pacientes com DC não têm de se submeter a uma dieta específica, nem existem fatores dietéticos conhecidos que agravem ou causem um aumento da atividade da doença, onde por sua vez, a dieta recomendada consiste numa dieta equilibrada focada no aporte energético, protéico, de vitaminas, minerais, e fluidos adequados (OLIVEIRA et al., 2017).

Segundo a literatura não existem alimentos específicos a evitar por todos os pacientes, mas alguns indivíduos podem ter intolerâncias alimentares ou desconforto com determinados alimentos, sendo que nesses casos esses alimentos devem ser evitados. Alguns alimentos ligados à exacerbação de sintomas incluem a lactose, sacarose, glúten, polióis (sorbitol -manitol), baixa ingestão de fruta e vegetais, baixa ingestão de fibras, ingestão de carne vermelha, consumo de álcool, ómega 6/ómega 3 desapropriado e ingestão de vitamina D insuficiente (KOTZE, 20II).

Desta forma, a terapia nutricional na fase de remissão passa por remover alimentos ou ingredientes que possam provocar sintomatologia, ou aos quais os pacientes sejam intolerantes, sendo importante substituir estes por outros alimentos que providenciem os 
mesmos nutrientes. Na fase aguda, deverão ser adotadas estratégias que revertam a sintomatologia, nomeadamente a evicção de cafeína, de bebidas alcoólicas e de fibra insolúvel, optando por fibra solúvel, e a suplementação com lactase ou a ingestão de produtos sem lactose (OLIVEIRA et al., 2017).

\section{MACRONUTRIENTES E ENERGIA}

As necessidades energéticas e proteicas dependem da gravidade e fase da doença, onde as necessidades energéticas nestes pacientes não estão necessariamente aumentadas, a menos que seja necessário um aumento ponderal. A recomendação energética indica um aporte de $25-30 \mathrm{kCal} / \mathrm{Kg}$ de peso ideal/dia e alguns estudos mostram que um aporte energético de $30-45 \mathrm{kCal} / \mathrm{Kg}$ de Nutrição Enteral (NE) está associado a uma taxa de remissão superior . Já as necessidades proteicas podem estar aumentadas, uma vez que a inflamação e o tratamento com corticosteroides induzem um balanço negativo de nitrogénio e provocam perda de massa magra, além de que em áreas de mucosa intestinal inflamada e ulcerada também ocorrem perdas protéicas (SUSKIND et al., 2014).

De forma a manter um balanço positivo de nitrogénio, recomenda-se 1,3 a 1,5 $\mathrm{g} / \mathrm{Kg} /$ dia de proteína, sendo que a European Society for Parenteral and Enteral Nutrition (ESPEN) nas suas orientações recomenda um aporte protéico de 1,2-1,5 g/ Kg/dia nos adultos e de $\mathrm{I} / \mathrm{Kg} /$ dia na fase de remissão. Relativamente aos indivíduos desnutridos e com má absorção, estes não devem exceder os $20 \%$ do aporte energético total sob a forma de lipídios. Existem evidências científicas que afirmam que o consumo de ácidos gordos ómega-3 está associado à diminuição da inflamação e manutenção da fase de remissão, através dos seus mediadores lipídicos. Em relação ao aporte de hidratos de carbono, o excessivo fornecimento de Oligossacáridos, Dissacáridos, Monossacáridos e Polióis Fermentáveis (FODMAPs), poderá ser um fator de suscetibilidade subjacente à DC, dado que a rápida fermentação dos mesmos no intestino induz ao aumento de permeabilidade intestinal . Esta hipótese defende que uma dieta com redução de (FODMAPs) reduz a sintomatologia gastrointestinal, no entanto, podem ocorrer alteração e diminuição da microbiota intestinal nos pacientes com DII, que por si já possuem um risco acrescido de disbiose (BRASPEN, 2019). 
Contudo, são necessárias mais pesquisas para determinararmos o quão rigorosa deverá de ser a restrição para providenciar resultados favoráveis a longo prazo, sendo que estes estudos têm de considerar fatores para além da sintomatologia, como a evolução da cicatrização da mucosa. No que diz respeito ao aporte de fibra, pacientes diagnosticados com DC não necessitam nem de restringir a ingestão de fibra, nem de aumentar acima dos níveis recomendados para a população saudável. A quantidade de fibra tolerada varia entre os indivíduos e pode também variar ao longo da evolução da patologia, pelo que é necessário regular o seu consumo ao longo de todo o tratamento (DIESTEL et al., 2012).

\section{MICRONUTRIENTES}

Ao nível das vitaminas, é comum o défice de ácido fólico (induzida também pela farmacoterapia com sulfassalazina) e de vitamina $B_{12}$, uma vez que as lesões mais comuns ocorrem no íleo e este é o local de absorção destas vitaminas. Assim, a suplementação de vitaminas poderá ser necessária, especialmente de vitamina $\mathrm{B} 6, \mathrm{~B}_{12}$ (sobretudo se houver receção do íleo) e ácido fólico, tal como de vitaminas lipossolúveis uma vez que doentes com malabsorção estão em risco de deficiências destas vitaminas. As carências vitamínicas podem-se manifestar de diversas formas, como cabelo e unhas secos e quebradiços, alterações dermatológicas, apatia, entre outros (OLIVEIRA et al., 2017).

Para reverter esta situação, em caso da má absorção de vitaminas lipossolúveis, recomenda-se a suplementação com triglicéridos de cadeia média (TCMs), que funcionam como veículo na absorção de nutrientes lipossolúveis. Em relação às vitaminas hidrossolúveis, a sua suplementação pode ser um dos métodos de tratamento do seu défice e, no caso dos doentes em fase de remissão, podem-se integrar gradualmente, e de acordo com a tolerância, os alimentos ricos nestes micronutrientes. A reposição de minerais poderá ser necessária devido a má digestão, má absorção, perdas hematológicas, interação fármaco-nutriente e baixa ingestão alimentar. Além disso, a diarreia aumenta a perda de zinco, potássio e selénio. Por sua vez, a carência de ferro heme é comum devido às perdas de sangue agudas ou crónicas nestes doentes, devendo ser suplementado em caso de deficiência, onde há carências de cálcio e vitamina $\mathrm{D}$ são recorrentes, estando os pacientes especialmente em risco, uma vez que os laticínios (Leites e derivados) são frequentemente 
evitados devido a intolerâncias e a absorção de vitamina $\mathrm{D}$ está comprometida (OLIVEIRA et al., 2017).

É importante a monitorização (e suplementação se necessário) dos níveis de cálcio sérico e de vitamina $\mathrm{D}$ em pacientes, tanto adultos como crianças, em fase ativa da patologia e pacientes tratados com esteróides, com vista à prevenção da osteopenia e osteoporose. Se o tratamento com esteroides for superior a 12 semanas, deve ser garantido um aporte de Iooo-I50o mg de cálcio/dia, recorrendo-se a suplementação se a dieta não for adequada, e 8oo UI de vitamina D. Além do défice destes micronutrientes, o déficit de magnésio, cobre, cromo, manganês e molibdénio também pode ser característico destes pacientes, pelo que se deve garantir o consumo das Dietary Reference Intakes (DRIs) para cada vitamina e mineral e caso não seja suficiente, recorrer a suplementação conforme a literatura científica (WEDRYCHOWICZ et al.,2016).

\section{ESTUDO DE CASO}

Paciente L.F.F.G, is anos, sexo femino de cor branca, sem antecedentes familiares de doença autoimune, iniciou dor abdominal, diarreia e perda ponderal aos io anos, onde após or mês do início, realizou endoscopia digestiva alta que evidenciou duodenite crônica inespecífica leve e colonoscopia com úlceras profundas entremeadas de áreas de mucosa normal, e alguns segmentos com lesões coalescentes com estreitamento da luz. Biópsia revelou colite crônica inespecífica moderada, que devido ao quadro clínico, endoscópico e histológicos compatíveis com Doença de Crohn, foi iniciado tratamento com prednisolona $2 \mathrm{mg} / \mathrm{kg} / \mathrm{dia}$ e mesalazina $20 \mathrm{mg} / \mathrm{kg} / \mathrm{dia}$.

A paciente foi encaminhada pelo Nutricionista ao HGF para uma consulta com o Gastroenterologista, onde na primeira consulta, informou que seu índice de massa corporal (IMC) de $18,56 \mathrm{~kg} / \mathrm{m}^{2}$, peso atual $41,76 \mathrm{~kg}$ e estatura $150 \mathrm{~cm}$ e abcesso e fístula perianais e que já encontravasse com Dieta oral restritiva com pouca fibra alimentar e com suporte nutricional evitando o aparecimento de sinais e sintomas clínicos.

Nos exames bioquímicos: hemoglobina 9,9g/dL; hematócrito 37\%; leucócitos 10.390/mm3; plaquetas $739.000 / \mathrm{mm} 3$; e proteína C-reativa $99,4 \mathrm{mg} / \mathrm{dL}$. Foi introduzido infliximabe na dose de $4 \mathrm{mg} / \mathrm{kg} /$ dose a cada o6 semanas; mesalazina teve a dose ajustada para 
$40 \mathrm{mg} / \mathrm{kg} / \mathrm{dia}$. Foram administrados metronidazol e ciprofloxacino - estes dois últimos devido ao abcesso perianal. Nos o6 meses seguintes, houve melhora de todo o quadro clínico, após I ano, repetiu colonoscopia, que era igual à inicial, e foi introduzida azatioprina na dose de $2 \mathrm{mg} / \mathrm{kg} / \mathrm{dia}$. Foi feita Ressonância de abdômen com fístulas ênterocólica e fístula anorretal. Tentou-se indução de remissão de atividade de doença com Nutrição Enteral exclusiva, porém a paciente recusou por sonda nasogástrica,concordando com a Nutrição via Oral. Por isso, foi mantida com infliximabe, mesalazina e azatioprina. Após 2 anos do diagnóstico, por apresentar quadro de distensão abdominal, saciedade precoce, dor abdominal, foi realizada ressonância de abdômen com estenose de íleo distal e, então, optou-se por laparotomia, que evidenciou múltiplas áreas de fístulas e estenoses, as quais foram ressecadas. Evoluiu com melhora nos o6 meses seguintes; porém, após 03 meses, iniciou anemia, perda de peso, sendo aumentado infliximabe $10 \mathrm{mg} / \mathrm{kg}$ a cada 04 semanas.

\section{RESULTADOS E DISCUSSÃO}

As doenças inflamatórias intestinais são crônicas e com pico de incidência entre is e 35 anos. São representadas por três doenças: retocolite ulcerativa, doença de Crohn e colite não classificada. As duas primeiras têm características típicas, e a terceira apresentase de forma que não permite diferenciar entre doença de Crohn e retocolite ulcerativa, sendo necessário o acompanhamento a longo prazo para esta definição.Nos Estados Unidos da América, a incidência é cerca de 4,5 em roo mil habitantes para Doença de Crohn e 2,4 em roo mil para retocolite ulcerativa sem dados estimados no Brasil, com proporções semelhantes em ambos os sexos.

Nota-se número crescente de diagnósticos nos últimos anos, alertando para a necessidade de o Nutricionista e o Gastroenterologista suspeitar e reconhecer uma doença inflamatória intestinal. As crianças têm doença mais grave que os adultos, o que afeta sobremaneira a qualidade de vida, não só pelas manifestações da doença como também pelos efeitos da terapêutica. A etiologia é a combinação de genética, meio ambiente e fatores imunológicos. Parentes de primeiro grau tem 20 vezes mais chance de desenvolver a doença que a população geral, onde além deste, outros dados reforçam a herança genética da doença, como a concordância da localização e tipo em uma mesma família. 
A origem genética da Doença de Crohn baseia-se em alterações em genes responsáveis pela homeostase celular, pela regulação da resposta imunológica e pela barreira adaptativa. Fatores ambientais que alteram a microbiota intestinal, como tipo de parto, condições sanitárias, alimentação inadequada, uso de antibióticos e tabagismo, são desencadeantes. Enquanto a retocolite ulcerativa acomete apenas a mucosa do cólon, a Doença de Crohn acomete, de forma transmural, qualquer área do trato gastrintestinal, sendo que as mais comumente afetadas são íleo terminal e cólon direito, e, em cerca de $50 \%$ dos casos, há também acometimento do trato gastrintestinal superior. O acometimento da doença determina as ulcerações profundas, de extensão descontínua, formando um aspecto típico à colonoscopia, chamado "pedra de calçamento", e também as complicações como estenoses e fístulas.As manifestações clínicas da Doença de Crohn incluem dor abdominal, diarreia, hematoquezia, inapetência, perda de peso, febre e lesão perianal, além de manifestações extraintestinais.

Cerca de metade dos pacientes tem baixa estatura e atraso puberal, que podem preceder os sintomas intestinais. O diagnóstico da Doença de Crohn é baseado no somatório de quadro clínico, alimentação, exames laboratoriais e exames endoscópicos com biópsia. Outro aspecto importante no diagnóstico é o exame de imagem do trato gastrintestinal superior, que, quando acometido, sugere necessidade de cirurgia precoce. $\mathrm{O}$ infliximabe é um anticorpo quimérico monoclonal contra o fator de necrose tumoral alfa (TNF- $\alpha$ ), efetivo em induzir e manter remissão da doença, porém seu efeito a longo prazo pode ser complicado pela perda de resposta que ocorre em $13 \%$ dos pacientes, podendo, então, o adalimumabe (anticorpo monoclonal humano contra TNF- $\alpha$ ) ser uma alternativa.

Quando o paciente já se apresenta com quadros de estenose e doença perianal ao diagnóstico, estas medicações têm resultados insuficientes. Estudos recentes demonstram que fenótipos fibroestenosantes e fistulizantes de Doença de Crohn não respondem bem à terapia medicamentosa e têm benefício com cirurgia precoce.

\section{CONCLUSÃO}

A Doença de Crohn é um importante causa de morbimortalidade, levando em consideração sua alta capacidade debilitante e a possibilidade de desenvolver inúmeras 
sequelas, inclusive fatais. Ainda não se sabe, na totalidade, os mecanismos que levam ao seu desenvolvimento, mas acredita-se que se trata de uma doença multifatorial, onde a associação entre fatores genéticos, ambientais e imunológicos culminam na a inflamação transmural do cólon. Novos estudos e investigações são necessários para melhor esclarecer estes mecanismos, principalmente no que diz respeito aos principais sintomas como: dor abdominal, diarreia crônica e febre, sendo que pode haver inúmeras manifestações extra intestinais. Os tratamentos convencionais, juntamente com a terapia nutricional têm sido parcialmente bem sucedidos ao reduzirem as crises agudas e prolongando a remissão da patologia, que neste momento limita a vida diária da paciente, bem como suas complicações, onde a complexidade de seu diagnóstico é um fator que implica no processo de tratamento que é fundamental para sua qualidade de vida.

\section{REFERÊNCIAS}

ABCD. Associação Brasileira de Colite Ulcerativa e Doença de Crohn. Biblioteca Virtual em Saúde do Ministério da Saúde,BVS, 2009.

Brazilian Society of Parenteral and Enteral Nutrition- BRASPEN J 2019; 33 (Supl).

CURY, D. B.; MOSS, A. C. Doenças inflamatórias intestinais: Retocolite Ulcerativa e Doença de Crohn. 2- ed. Rio de Janeiro: Rubio, 2015.

DIESTEL, CF, SANTOS, MC dos, ROMI, MD. Tratamento Nutricional nas Doenças Inflamatórias Intestinais. Rev do Hosp Univ Pedro Ernesto, UERJ. 2012;52-8.

FLORA, APL, Dichi I. Aspectos atuais na terapia nutricional da Doença Inflamatória Intestinal. Rev Bra Nutr Clin. 2005; 2I (2):131-I37.

LOMER, MCE. Symposium 7: Nutrition in inflammatory bowel disease Dietary and nutritional considerations for inflammatory bowel disease.Proceeding sof the Nutrition Society. 2011; 70:329-335. 
OliveIRA, C. ANTUNES, C. SANTOS,C.MARQUES, A.SOUZA, M. Suporte Nutricional na Doença de Crohn. ACTA PORTUGUESA DE NUTRIÇÃO io (2017) 44-48 | LICENÇA: cc-by-nc | http://dx.doi.org/ı.210II/apn.2017.1007.

KOTZE LMS; KOTZE PG; KOTZE LR. Doença de Crohn. In: DANI R; PASSOS MCF. Gastroenterologia Essencial. Rio de Janeiro: Guanabara Koogan, 201r. p. $347-379$.

SUSKIND, D.L. WAHBEH, G. GREGORY, N. VENDETTUOLI, H. CHRISTIE, D. Nutritional therapy in pediatric Crohn disease: the specific carbohydrate diet. J Pediatr Gastroenterol Nutr. 2014;58:87-91. https://doi.org/10.1097/MPG.oooooooooooooro3.

WEDRYCHOWICS A, ZAJAC A, TOMAZIK P. Advances in nutritional therapy in inflammatory bowel diseases: Review. World J Gastroenterol. 2016;22(3):1045-66. 